\title{
Temozolomide Alone or Combined with Capecitabine for the Treatment of Advanced Pancreatic Neuroendocrine Tumor
}

\author{
Louis de Mestier $^{\mathrm{a}}$ Thomas Walter $^{\mathrm{b}}$ Camille Evrard $^{\mathrm{c}}$ Paul de Boissieu $^{\mathrm{d}}$ \\ Olivia Hentic ${ }^{a}$ Jérôme Cros ${ }^{e}$ David Tougeron ${ }^{f}$ Catherine Lombard-Bohas ${ }^{b}$ \\ Vinciane Rebours $^{\text {a }}$ Pascal Hammel $^{9}$ Philippe Ruszniewski ${ }^{a}$ \\ a Department of Pancreatology and Gastroenterology, ENETS Centre of Excellence, Beaujon Hospital, and \\ Paris University, Clichy, France; ${ }^{b}$ Department of Digestive Oncology, ENETS Centre of Excellence, Edouard \\ Herriot Hospital, Lyon, France; ${ }^{C}$ Department of Medical Oncology, Poitiers University Hospital, Poitiers, France; \\ ${ }^{\mathrm{d}}$ Department of Epidemiology and Public Health, Le Kremlin-Bicêtre Hospital, Le Kremlin-Bicêtre, and Paris-Sud \\ University, Le Kremlin-Bicêtre, France; ${ }^{e}$ Department of Pathology, ENETS Centre of Excellence, Beaujon Hospital, \\ and Paris University, Clichy, France; ${ }^{f}$ Department of Gastroenterology, Poitiers University Hospital, Poitiers, France; \\ ${ }^{g}$ Department of Digestive Oncology, ENETS Centre of Excellence, Beaujon Hospital, and Paris University, Clichy, \\ France
}

\section{Keywords}

Neuroendocrine tumor · Metastases · Chemotherapy ·

Temozolomide $\cdot$ Capecitabine $\cdot$ Pancreas

\begin{abstract}
Background: The combination of capecitabine (CAP) with temozolomide (TEM) chemotherapy in advanced pancreatic neuroendocrine tumors (PanNET) relies on limited evidence. We compared TEM-CAP to TEM alone in patients with advanced PanNET. Methods: Consecutive patients with advanced PanNET treated with TEM or TEM-CAP between 2004 and 2017 in three expert centers were included. Progressionfree survival (PFS), tolerance, tumor response, and overall survival were compared between the two groups. Propensity-based analyses were performed to reduce confounding bias due to the nonrandomized setting. Results: TEM and TEM-CAP were administered to 38 patients and 100 patients, respectively, with a median age of 58 years. The patients in the TEM group more often had hormonal syndromes $(p=$
\end{abstract}

$0.03)$, a longer median delay to diagnosis $(p=0.001)$, and a higher number of pretreatment lines $(p<0.001)$. The performance status was 0 in $58 \%$ versus $65 \%$ of the patients, and tumor's median $\mathrm{Ki}-67$ index was $8 \%$ versus $11 \%$, respectively. Tolerance was similar, except that there were more cases of asthenia in the TEM group $(p=0.017)$ and more cases of hand-foot syndrome in the TEM-CAP group $(p=0.025)$. The objective response rate was $34 \%$ versus $51 \%(p=0.088)$. The raw median PFS was similar with TEM and with TEM-CAP (21.4 vs. 19.8 months, $p=0.84$ ). Although CAP tended to decrease the risk of progression in Cox multivariate analysis (HR $0.65, p=0.12$ ), it had no effect after adjustment for the propensity score (HR 1.06, $p=0.80$ ). Conclusions: TEM-CAP might not prolong PFS but might achieve a higher response rate than TEM alone. Hence, TEM-CAP might be preferred when tumor shrinkage is the main therapeutic objective. Otherwise, TEM might be adequate for patients with an impaired performance status or in case of extrahepatic metastases.

(c) 2019 The Author(s)

Published by S. Karger AG, Basel

\begin{tabular}{|c|c|c|}
\hline KARGER & $\begin{array}{l}\text { () } 2019 \text { The Author(s) } \\
\text { Published by S. Karger AG, Basel }\end{array}$ & $\begin{array}{l}\text { Karger } \\
\text { Open access }\end{array}$ \\
\hline $\begin{array}{l}\text { ger@karger.com } \\
\text { er.com/nen }\end{array}$ & $\begin{array}{l}\text { This article is licensed under the } \\
\text { NonCommercial-NoDerivatives } 4 \\
\text { NC-ND) (http://www.karger.con } \\
\text { Usage and distribution for comme } \\
\text { tribution of modified material requ }\end{array}$ & $\begin{array}{l}\text { Commons Attribution- } \\
\text { ational License (CC BY- } \\
\text { es/OpenAccessLicense). } \\
\text { rposes as well as any dis- } \\
\text { tten permission. }\end{array}$ \\
\hline
\end{tabular}

Dr. Louis de Mestier, MD

Department of Pancreatology and Gastroenterology

ENETS Centre of Excellence, Beaujon Hospital, and Paris University

100 boulevard du Général Leclerc, FR-92110 Clichy (France)

E-Mail louis.demestier@aphp.fr 


\section{Introduction}

Well-differentiated pancreatic neuroendocrine tumors (PanNET) are relatively rare neoplasms, but their incidence is increasing [1]. Distant metastases occur in more than $50 \%$ of patients [2]. Chemotherapy remains the cornerstone of the therapeutic armamentarium for advanced PanNET. It can particularly benefit patients with aggressive tumors (i.e., progressive tumors, with a high tumor burden and/or high Ki-67 index) or in a neoadjuvant setting to achieve tumor shrinkage and thus allow secondary tumor resection $[2,3]$.

Among the recommended chemotherapy regimens, the combination of capecitabine (CAP) with temozolomide (TEM) has been widely used. The efficacy of the TEM-CAP combination in patients with metastatic PanNET has been well documented, with an objective response rate (ORR) around $40-50 \%$ and a median progression-free survival (PFS) of about 12-15 months [4-8]. However, whether the addition of CAP to TEM provides increased efficacy compared to TEM alone has not yet been demonstrated [7, 9]. Recently, Campana et al. [8] reported that TEM-CAP was not superior to TEM as a single agent in terms of PFS or overall survival (OS), but that it provided a higher ORR. Conversely, the preliminary results of a prospective randomized phase II study comparing TEM-CAP with TEM reported prolonged PFS and OS but a similar ORR [10]. As discussed in this paper, those preliminary results were somehow surprising and might have been influenced by an imbalance between the patients included, as those in the TEM-CAP group had PanNET of a lower grade. Besides, TEM-CAP could cause increased toxicity compared to TEM alone [10].

We aimed to compare the efficacy of and tolerance to TEM-CAP versus TEM alone in patients with metastatic PanNET in a large multicenter series. In order to reduce confounding bias due to the nonrandomized setting, we performed our analyses based on a propensity score.

\section{Patients and Methods}

\section{Patients}

The records on all consecutive patients with PanNET who had received at least 1 cycle of TEM or TEM-CAP chemotherapy between July 2004 and December 2017 in three French expert centers (Beaujon University Hospital, Clichy; Edouard Herriot University Hospital, Lyon; and Poitiers University Hospital, Poitiers) were retrospectively studied. All patients had a pathologically proven, well-differentiated PanNET. Patients with a NET arising from another origin (including an unknown primary) or a poorly differentiated neuroendocrine carcinoma were not included.
Chemotherapy Administration and Patient Management

TEM alone was administered during 5 days every 28 days. The TEM-CAP regimen consisted of oral administration of CAP (750 $\mathrm{mg} / \mathrm{m}^{2}$ ) twice daily during days 1-14 and TEM once daily during days $10-14$, every 28 days. In both regimens, TEM was started at a dose of $150 \mathrm{mg} / \mathrm{m}^{2}$ in cycle 1 . Depending on the patient's tolerance and investigator's decision, it could be increased to $200 \mathrm{mg} / \mathrm{m}^{2}$ for the following cycles.

All patients underwent a contrast-enhanced thoracoabdominopelvic CT scan within 6 weeks before the initiation of chemotherapy. A clinical, biological, and CT scan evaluation was performed every 3-4 cycles, and the imaging data were systematically reviewed by experienced radiologists during weekly NET-dedicated multidisciplinary tumor boards.

\section{Data Collection}

Demographic, clinical, and survival data were retrieved from the medical records. Tolerance was assessed according to the highest toxicity grade following the National Cancer Institute Common Toxicity Criteria (version 4.0). The pathological samples were all analyzed at high-volume TENPATH (French NET-dedicated pathology network) centers for morphology and Ki-67 immunostaining. In case of multiple pathological specimens, the most recent before chemotherapy introduction was selected for this purpose. Grading was performed retrospectively following the 2017 WHO classification (G1 with a Ki-67 index $<3 \%$, G2 with a Ki-67 index of 3-20\%, and G3 with a Ki-67 index >20\%) [11].

\section{Statistical Analysis}

Continuous variables are described as means (standard deviations) and compared using Student's $t$ test or the Mann-Whitney test. Qualitative variables are described as frequencies (percentages) and compared using the $\chi^{2}$ test or Fisher's exact test.

The primary endpoint analyzed was PFS. Secondary endpoints were toxicity, ORR, and OS. Tumor response was assessed according to the RECIST 1.0 or RECIST 1.1 criteria depending on the period of management of the patients $[12,13]$. The ORR was defined as the sum of the rates of complete response and partial response as the best response. The disease control rate was calculated as the sum of the ORR and the stability rate. PFS was measured from the date of the first chemotherapy cycle to the date of progression or death, or censored at the date of last news. OS was measured from the date of the first chemotherapy cycle to the date of death, or censored at the date of last news. The cutoff date was set at June 1, 2018. Median PFS and median OS were estimated using the Kaplan-Meier method. The 95\% confidence intervals (CI) for median PFS and OS were derived from log hazard. Survival rates were compared using the log-rank test. Factors associated with PFS and OS were explored using Cox regression models, and factors associated with tumor response were assessed using a logistic regression model. Each time, all relevant baseline variables were entered into a univariate model, and then all noncollinear variables with $p$ values $<0.20$ were entered into a multivariate model. All analyses were two-sided. Any $p$ value $<0.05$ was considered significant. Analyses were performed with SPSS ${ }^{\circledR}$ (version 20; IBM) and SAS (version 9.4; SAS Institute) software.

\section{Propensity Score Analyses}

In order to reduce confounding bias due to the nonrandomized setting of the study, a propensity score was used [14]. It aimed to 
Table 1. Baseline clinical and pathological characteristics of the 138 patients with advanced PanNET treated with TEM or TEM-CAP

\begin{tabular}{|c|c|c|c|}
\hline & $\begin{array}{l}\text { TEM } \\
(n=38)\end{array}$ & $\begin{array}{l}\text { TEM-CAP } \\
(n=100)\end{array}$ & $p$ value \\
\hline Male gender, $n(\%)$ & $19(50)$ & $58(58)$ & 0.40 \\
\hline Age, years & $59.5 \pm 11.6$ & $57.0 \pm 12.1$ & 0.53 \\
\hline Hormonal syndrome, $n(\%)$ & $12(31.6)$ & $15(15.0)$ & 0.03 \\
\hline Gastrinoma & 5 & 8 & \\
\hline Insulinoma & 1 & 3 & \\
\hline Glucagonoma & 4 & 3 & \\
\hline VIPoma & 1 & 0 & \\
\hline Parathyroid hormone-related protein & 1 & 0 & \\
\hline Calcitonin & 0 & 1 & \\
\hline $\mathrm{Ki}-67$ index, $\%^{\mathrm{a}}$ & $7.8 \pm 6.2$ & $11.0 \pm 10.3$ & 0.16 \\
\hline Tumor grade, $n(\%)^{\mathrm{a}}$ & & & 0.17 \\
\hline G1 & $6(15.8)$ & $12(12.5)$ & \\
\hline G2 & $31(81.6)$ & $71(74)$ & \\
\hline G3 & $1(2.6)$ & $13(13.5)$ & \\
\hline WHO performance status, $n(\%)$ & & & 0.51 \\
\hline 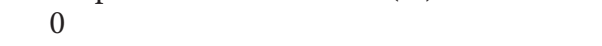 & $22(57.9)$ & $65(65)$ & \\
\hline 1 & $14(36.8)$ & $33(33)$ & \\
\hline 2 & $2(5.3)$ & $2(2)$ & \\
\hline Tumor stage, $n(\%)$ & & & 0.83 \\
\hline Locally advanced & $1(2.6)$ & $3(3)$ & \\
\hline Liver metastases only & $22(57.9)$ & $52(52)$ & \\
\hline Extrahepatic metastases & $15(39.5)$ & $45(45)$ & \\
\hline Progression within 12 months, $n(\%)$ & $\begin{array}{l}33 / 35(94.3) \\
3 \text { nonevaluable }\end{array}$ & $\begin{array}{l}69 / 70(98.6) \\
30 \text { nonevaluable }\end{array}$ & 0.26 \\
\hline Delay to diagnosis, months & $58.7 \pm 49.6$ & $29.9 \pm 35.7$ & 0.001 \\
\hline \multicolumn{4}{|l|}{ Previous treatments, $n(\%)$} \\
\hline Primary tumor surgery & $21(55.3)$ & $51(51)$ & 0.65 \\
\hline Chemotherapy & $16(42.1)$ & $22(22)$ & 0.02 \\
\hline Targeted therapy & $10(26.3)$ & $14(14)$ & 0.09 \\
\hline Liver transarterial (chemo-)embolization & $7(18.4)$ & $8(8)$ & 0.12 \\
\hline Peptide receptor radionuclide therapy & $2(5.3)$ & $1(1)$ & 0.18 \\
\hline Somatostatin analog treatment & $19(50.0)$ & $29(29)$ & 0.02 \\
\hline Previous lines, $n$ & & & $<0.001$ \\
\hline 0 & $9(23.7)$ & $58(58)$ & \\
\hline$\geq 1$ & $29(76.3)$ & $42(42)$ & \\
\hline
\end{tabular}

Values are presented as $n(\%)$ or mean \pm SD. Bold type denotes significance. PanNET, pancreatic neuroendocrine tumor; TEM, temozolomide; CAP, capecitabine. ${ }^{*} 4$ missing values.

predict the individual probability of receiving TEM-CAP rather than TEM conditional on the baseline characteristics. A logistic regression model, aiming to determine which baseline variables were associated with the type of chemotherapy, was used to build the propensity score. The accuracy of the model was measured by the area under the ROC curve and the Hosmer-Lemeshow adequacy test. A Cox regression model adjusted for the propensity score was computed to explore whether the chemotherapy type influenced the risk of progression (or death), and a logistic regression model adjusted for the propensity score was used to explore whether the chemotherapy type influenced the tumor response.
Then, two other methods were used for sensitivity analyses for PFS, which was the primary endpoint. First, patients of both groups were matched (1:1) following their propensity score. To assess their comparability, the standardized differences in baseline variables between the matched groups were computed [15]. PFS was compared between the matched groups using the Kaplan-Meier method and the log-rank test. Finally, PFS was analyzed using the inverse probability treatment weighting (IPTW) method, where each patient is individually weighted by a synthetic propensity score, namely, " $1 /$ score" for patients treated with TEM-CAP and " $1 /(1-$ score $)$ " for patients treated with TEM. 
Table 2. Multivariate logistic regression model of variables associated with the administration of TEM-CAP rather than TEM alone, used to build the propensity score

\begin{tabular}{llc}
\hline & OR (95\% CI) & $p$ value \\
\hline Hormonal syndrome (vs. absence) & $0.45(0.17-1.21)$ & 0.12 \\
Ki-67 index (each additional 1\%) & $1.05(0.99-1.12)$ & 0.09 \\
Delay to initial diagnosis (each additional month) & $0.99(0.98-1.12)$ & 0.16 \\
Previous chemotherapy administration (vs. absence) & $0.30(0.12-0.76)$ & $\mathbf{0 . 0 1}$ \\
Previous somatostatin analog administration (vs. absence) & $0.66(0.25-1.71)$ & 0.39 \\
\hline
\end{tabular}

Bold type denotes significance. TEM, temozolomide; CAP, capecitabine.

Table 3. Main adverse events and their severity in 132 patients with advanced PanNET treated with TEM or TEM-CAP

\begin{tabular}{lclllll}
\hline & \multicolumn{2}{l}{ TEM $(n=38)$} & & \multicolumn{2}{l}{ TEM-CAP $(n=94)$} & \multirow{2}{*}{$p$ value } \\
\cline { 2 - 3 } & grade 1-2 & grade 3-4 & & grade 1-2 & grade 3-4 & \\
\hline Nausea/vomiting & $15(39.5)$ & $1(2.6)$ & & $42(44.7)$ & $2(2.1)$ & 0.86 \\
Diarrhea & $4(10.5)$ & 0 & & $9(9.6)$ & $2(2.1)$ & 0.66 \\
Neutropenia & $2(5.3)$ & $2(5.3)$ & & $13(13.8)$ & $6(6.4)$ & 0.35 \\
Thrombocytopenia & $6(15.8)$ & $5(13.2)$ & & $12(12.8)$ & $12(12.8)$ & 0.89 \\
Anemia & $6(15.8)$ & 0 & & $16(17)$ & $1(1.1)$ & 0.80 \\
Stomatitis & $2(5.3)$ & 0 & $3(3.2)$ & 0 & 0.63 \\
Asthenia & $18(47.4)$ & $3(7.9)$ & & $41(43.6)$ & 0 & $\mathbf{0 . 0 1 7}$ \\
Hand-foot syndrome & 0 & 0 & $13(13.8)$ & $3(2.3)$ & $\mathbf{0 . 0 2 5}$ \\
\hline
\end{tabular}

Bold type denotes significance. PanNET, pancreatic neuroendocrine tumor; TEM, temozolomide; CAP, capecitabine.

\section{Results}

\section{Patients}

Overall, 138 patients were included; 38 and 100 were treated with TEM alone and TEM-CAP, respectively. The mean total follow-up time after chemotherapy initiation was $43.4 \pm 26.8$ months in the TEM group and $33.4 \pm 21.7$ months in the TEM-CAP group ( $p=0.053)$. Some imbalance in the baseline characteristics existed between the two groups (Table 1). For instance, patients treated with TEM alone more frequently had a functioning PanNET ( 31.6 vs. $15 \%, p=0.03$ ) and tended to have a lower Ki-67 index ( 7.8 vs. $11 \%, p=0.16)$. In addition, the delay to initial diagnosis was longer (58.7 vs. 29.9 months, $p<0.001$ ) and patients more frequently were pretreated (76.3 vs. $42 \%, p=0.0003$ ) in the TEM group, including more frequent previous chemotherapy ( 42.1 vs. $22 \%, p=0.02)$ and somatostatin analog treatment ( 50 vs. $29 \%, p=0.02$ ). Almost all patients of both groups had documented progression within the 12 previous months.

\section{Construction of the Propensity Score}

The variables that were imbalanced between the two groups (hormonal syndrome, Ki-67 index, delay to diagnosis, and previous administration of chemotherapy and/ or somatostatin analogs) were entered into a multivariate logistic regression model in order to calculate the propensity score for each patient (Table 2). The number of previous treatment lines was not used, because of collinearity with most of the other variables. The propensity score showed good accuracy, with an area under the ROC curve of 0.77 and a Hosmer-Lemeshow adequacy score of 0.66 .

\section{Chemotherapy Administration and Tolerance}

The patients treated with TEM or TEM-CAP received a mean number of $7.3 \pm 4.4$ and $7 \pm 4.5$ cycles, respectively $(p=0.78)$. The mean TEM dose per cycle was $177 \pm 42$ and $196 \pm 44 \mathrm{mg}$, respectively $(p=0.083)$, and $90 \%( \pm 24 \%)$ of the TEM-CAP cycles contained CAP.

Tolerance data were available for 132 patients. Grade 3-4 adverse events occurred in 9 patients (23.7\%) with
86

Neuroendocrinology 2020;110:83-91 DOI: $10.1159 / 000500862$ de Mestier et al. 
TEM versus 21 patients $(22.3 \%)$ with TEM-CAP $(p=1)$. The most frequent adverse events are shown in Table 3. Tolerance was similar globally, but TEM-CAP caused more hand-foot syndrome $(p=0.025)$ and less asthenia $(p=0.017)$ than did TEM.

\section{Progression-Free Survival}

In raw analysis, PFS was similar between patients treated with TEM-CAP (median 21.4 months, 95\% CI 12.5-27.4) and those treated with TEM-CAP (median PFS 19.8 months, 95\% CI 13.6-26.1; HR 0.96, 95\% CI $0.63-1.47, p=0.84$ ) (Fig. 1a). The presence of a hormonal syndrome, a higher Ki-67 index, an impaired WHO performance status, the presence of extrahepatic metastases, previous primary tumor resection, and previous chemotherapy were significantly and independently associated with an increased risk of progression (Table 4). In comparison to TEM alone, TEM-CAP was associated with a $35 \%$ decrease in the risk of progression (HR 0.65, $95 \%$ CI $0.37-1.12, p=0.12$ ), although this difference was not statistically significant.

In Cox regression analysis adjusted for the propensity score, TEM-CAP was not associated with a decreased risk of progression in comparison with TEM alone (HR 1.06, $95 \%$ CI $0.66-1.71, p=0.80)$. Patients were then matched $1: 1$ on their propensity score. For this analysis, 32 patients were included in each group. The baseline characteristics of each matched group were similar (online suppl. Table 1; see www.karger.com/doi/10.1159/000500862 for all online suppl. material). The risk of progression was not different between the TEM-CAP and the TEM group (HR $1.13,95 \%$ CI $0.64-2.00, p=0.67$ ) (Fig. 1b). Finally, PFS was compared using the IPTW method for all 138 patients. The risk of progression was not different between the TEM-CAP and the TEM group (HR 1.23, 95\% CI $0.74-2.06, p=0.43$ ) (Fig. 1c).

\section{Best Response to Chemotherapy}

Complete response, partial response, stability, and progression were observed in 5.3, 28.9, 39.5, and $26.3 \%$ of the patients treated with TEM, and in 2, 49, 36, and $13 \%$ of the patients treated with TEM-CAP, respectively ( $p=$ $0.087)$. The ORR ( 34.2 vs. $51 \%, p=0.088)$ and disease control rate ( 73.7 vs. $87 \%, p=0.075$ ) tended to be higher in the TEM-CAP group.

In multivariate analysis, an impaired $\mathrm{WHO}$ performance status and the presence of extrahepatic metastases were significantly and independently associated with a lower ORR (Table 5). TEM-CAP (in comparison to TEM alone) tended to increase the ORR, although the differ-

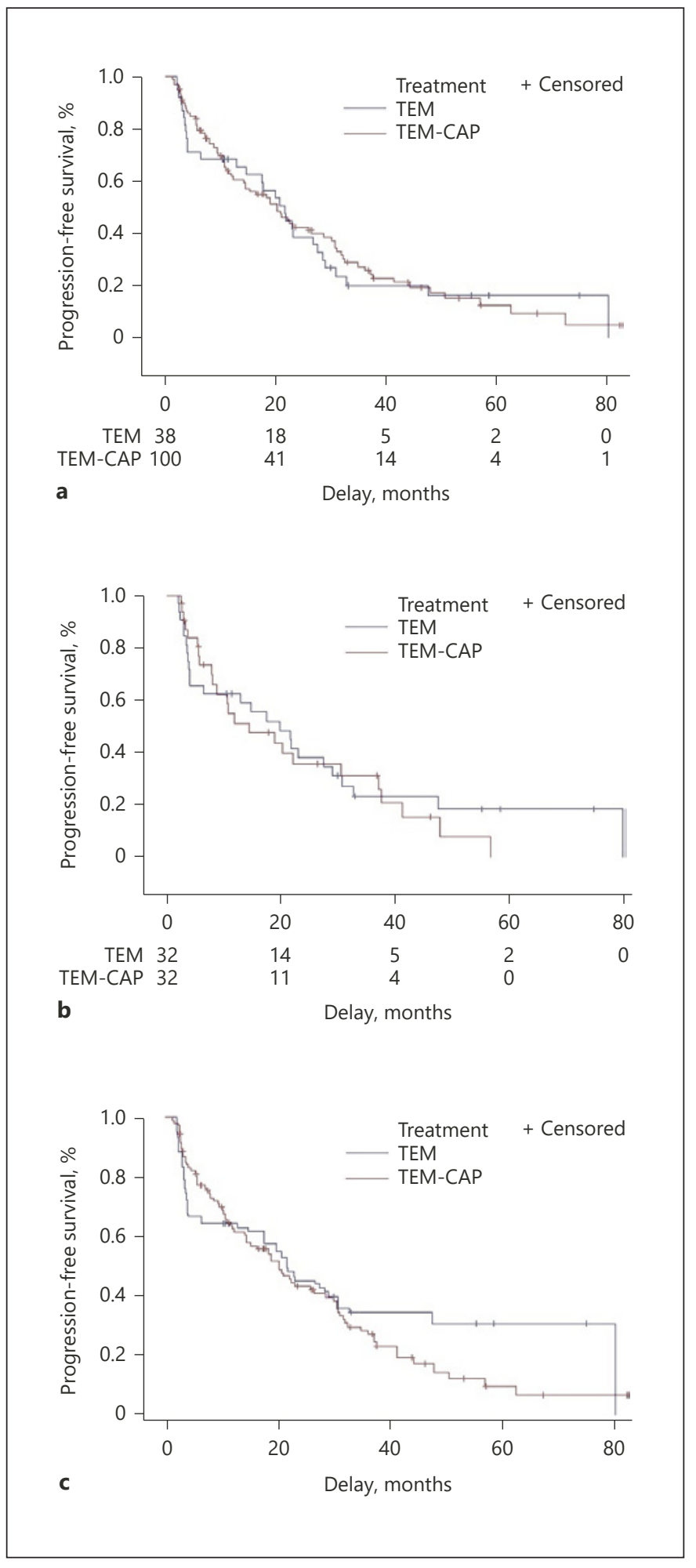

Fig. 1. Progression-free survival among 138 patients with advanced pancreatic neuroendocrine tumors treated with temozolomide (TEM) or TEM-capecitabine (TEM-CAP). a Raw analysis. b Analysis of patients matched for their propensity score. c Analysis using the inverse probability treatment weighting method. 
Table 4. Multivariate Cox regression analysis of variables associated with the risk of progression in the 138 patients with PanNET treated with TEM or TEM-CAP

\begin{tabular}{lll}
\hline & HR $(95 \% \mathrm{CI})$ & $p$ value \\
\hline TEM-CAP (vs. TEM alone) & $0.65(0.37-1.12)$ & 0.12 \\
Hormonal syndrome (vs. absence) & $1.89(1.09-3.29)$ & $\mathbf{0 . 0 2}$ \\
Ki-67 index (each additional 1\%) & $1.03(1.01-1.06)$ & $\mathbf{0 . 0 0 3}$ \\
WHO PS-1 or PS-2 (vs. WHO PS-0) & $2.27(1.40-3.66)$ & $\mathbf{0 . 0 0 1}$ \\
Extrahepatic metastases (vs. absence) & $1.88(1.20-2.93)$ & $\mathbf{0 . 0 0 6}$ \\
Delay to diagnosis (each additional month) & $1.00(0.99-1.01)$ & 0.23 \\
Primary tumor surgery (vs. absence) & $0.57(0.36-0.90)$ & $\mathbf{0 . 0 2}$ \\
Previous chemotherapy administration (vs. absence) & $1.79(1.08-2.96)$ & $\mathbf{0 . 0 3}$ \\
Previous somatostatin analog administration (vs. absence) & $1.39(0.83-2.34)$ & 0.21 \\
\hline
\end{tabular}

Bold type denotes significance. PanNET, pancreatic neuroendocrine tumor; TEM, temozolomide; CAP, capecitabine; PS, performance status.

Table 5. Multivariate logistic regression model of variables associated with objective response in the 138 patients with PanNET treated with TEM or TEM-CAP

\begin{tabular}{lll}
\hline & OR (95\% CI) & $\begin{array}{l}p \\
\text { value }\end{array}$ \\
\hline TEM-CAP (vs. TEM alone) & $2.28(0.91-5.69)$ & 0.077 \\
Ki-67 index (each additional 1\%) & $0.98(0.93-1.03)$ & 0.50 \\
WHO PS-1 or PS-2 (vs. WHO PS-0) & $0.21(0.08-0.51)$ & $\mathbf{0 . 0 0 1}$ \\
$\begin{array}{l}\text { Extrahepatic metastases (vs. absence) } \\
\text { Previous treatment lines }\end{array}$ & $0.45(0.22-0.90)$ & $\mathbf{0 . 0 2 4}$ \\
$\quad$ (each additional one) & $0.87(0.60-1.27)$ & 0.47 \\
\hline
\end{tabular}

Bold type denotes significance. PanNET, pancreatic neuroendocrine tumor; TEM, temozolomide; CAP, capecitabine; PS, performance status.

ence was not statistically significant (OR 2.28, 95\% CI $0.91-5.69, p=0.077)$, which remained similar after adjusting for the propensity score only (OR $2.02,95 \%$ CI $0.87-4.69, p=0.10)$.

\section{Overall Survival}

Twenty patients (52.6\%) of the TEM group and $26 \mathrm{pa}-$ tients $(26 \%)$ of the TEM-CAP group died during followup. The estimated median OS was 47.6 months (95\% CI 33.6-61.7) among the patients treated with TEM and 75.2 months (95\% CI 58.5-91.9) among those treated with TEM-CAP. The reduction in the risk of death in the TEM-CAP group tended to be significant (HR 0.66, 95\% CI $0.37-1.19, p=0.16$ ). Nevertheless, the risk of death was not statistically significantly different between the TEM and the TEM-CAP group in Cox regression multivariate
Table 6. Multivariate Cox regression analysis of variables associated with the risk of death in the 138 patients with PanNET treated with TEM or TEM-CAP

\begin{tabular}{lll}
\hline & HR (95\% CI) & $\begin{array}{l}p \\
\text { value }\end{array}$ \\
\hline TEM-CAP (vs. TEM alone) & $0.70(0.36-1.38)$ & 0.30 \\
Ki-67 index (each additional 1\%) & $1.04(1-1.08)$ & 0.065 \\
WHO PS-1 or PS-2 (vs. WHO PS-0) & $1.82(0.87-3.77)$ & 0.11 \\
$\begin{array}{l}\text { Extrahepatic metastases (vs. absence) } \\
\text { Previous treatment lines }\end{array}$ & $1.3(0.79-2.14)$ & 0.30 \\
$\quad$ (each additional one) & $1.48(1.14-1.92)$ & $\mathbf{0 . 0 0 3}$ \\
\hline
\end{tabular}

Bold type denotes significance. PanNET, pancreatic neuroendocrine tumor; TEM, temozolomide; CAP, capecitabine; PS, performance status.

analysis (HR 0.70, 95\% CI 0.36-1.38, $p=0.30$ ) (Table 6) and in Cox multivariate analysis adjusted for the propensity score (HR $0.67,95 \%$ CI $0.34-1.32, p=0.25$ ). Conversely, a higher number of previous treatment lines and a higher Ki-67 index - although the latter was not statistically significant - increased the risk of death.

\section{Discussion}

The TEM-CAP combination was associated with a similar PFS but a higher ORR compared to TEM alone in this large retrospective multicenter series of mostly pretreated patients with advanced, progressive PanNET. All 19 studies previously published about the efficacy of TEM with or without CAP in PanNET [4-6, 8, 16-30] were
88

Neuroendocrinology 2020;110:83-91 DOI: $10.1159 / 000500862$ de Mestier et al. 
retrospective, included not only patients with PanNET but also patients with NET from other origins, and reported on various treatments, mostly TEM-CAP but also TEM combined with thalidomide or bevacizumab $[7,9]$. When considering PanNET only, the ORRs ranged from 15 to $70 \%$ (average around $45 \%$ ) and the median PFS ranged from 6 to 20 months (average around 14 months) in these studies. These wide variations could be explained by the differences in the treatments used: as discussed by Campana et al. [8], the series with the highest ORR included PanNET patients uniformly treated with TEMCAP rather than with TEM alone.

The rationale of combining TEM and CAP relies on limited evidence. Fine et al. [6] reported a synergistic proapoptotic effect of these drugs in two NET cell lines in vitro. The cytotoxicity of TEM is mainly mediated by the $\mathrm{O}^{6}$-methylguanine adducts, which can mispair with thymine during DNA replication. The mismatch repair system, which is virtually proficient in all PanNET, removes and replaces the mismatched thymine by another thymine; these cycles of futile DNA processing induce cytotoxic double-strand DNA breaks that trigger apoptosis [7, 31]. $O^{6}$-methylguanine DNA methyltransferase (MGMT) removes $\mathrm{O}^{6}$-methylguanine adducts and is the main mechanism of NET resistance to TEM identified so far [7]. Indeed, MGMT deficiency is frequent in PanNET and partly explains the efficacy of TEM in this tumor type $[4,8,25]$. CAP, after conversion into 5 -fluorouracile by the liver, causes the incorporation of 5-FdUTP into the DNA. Additionally, one in vitro study showed that 5-fluorouracile reduced the MGMT protein content and, to a lesser extent, MGMT mRNA expression [32]. Among possible explanations, 5-FdUMP inhibits thymidylate synthase, which synthetizes dTMP from dUMP, thereby reducing thymidine pools. This may reduce the repair activity of MGMT, thereby potentiating the effects of TEM [5]. In addition, CAP-induced thymidine depletion may be amplified by DNA mismatch repair futile repetitive cycles in MGMT-deficient cells, leaving the cell without dTTP and causing DNA breaks [6]. These hypotheses may explain why a potential synergistic effect of TEM and CAP would rely on pretreatment with CAP followed by TEM. Indeed, this sequence was more effective in PanNET cell lines than exposition to CAP alone, TEM alone, or TEM followed by or simultaneously administered with CAP (which resulted in additive but not synergistic cytotoxicity) [6]. However, because TEM-CAP has limited efficacy in non-PanNET, the CAP-induced enhancement of TEM cytotoxicity may not be the single mechanism explaining the synergy between TEM and CAP $[4,8,21]$.
To our knowledge, among all the previous studies about the efficacy of TEM with or without CAP, only one has explored the influence of the type of chemotherapy (TEM or TEM-CAP) on the ORR and PFS [8]. In that retrospective series of 104 patients with advanced NET from various origins, TEM-CAP (vs. TEM alone) had no influence on the risk of progression (HR 0.93, 95\% CI $0.58-1.49, p=0.766$ ) as in our current study. However, any retrospective comparison exposes a selection bias with regard to why one patient was treated with TEMCAP and another with TEM alone. In our study, in Cox regression multivariate analysis, TEM-CAP tended to be associated with a decrease in the risk of progression (HR $0.65,95 \%$ CI $0.37-1.12, p=0.12$ ) in comparison with TEM alone. In order to further minimize the selection bias, we built a propensity score to more robustly predict an individual's probability of having been treated with TEM-CAP rather than with TEM. Interestingly, TEMCAP was associated with a PFS similar to that with TEM alone, whatever the methodology used (a Cox model adjusted for the propensity score, comparison of patients matched for their propensity score, and comparison using the IPTW method). In other words, PFS was similar in both groups considering the probability of receiving TEM-CAP being equal for each patient.

In addition, Campana et al. [8] reported that TEMCAP was associated with a higher ORR than was TEM (OR 3.87, $p=0.019$ ), although their multivariate analysis was only adjusted for MGMT promoter methylation. Likewise, we found that TEM-CAP tended to provide a higher ORR than did TEM alone (51 vs. $34.2 \%, p=0.088$ ), with an increased probability of objective response either in raw analysis (OR 2.28, $p=0.077$ ) or in analysis adjusted for the propensity score (OR 2.02, $p=0.10$ ). Those differences did not reach statistical significance, likely due to lack of power. Besides, having a WHO performance status of 0 and having no extrahepatic metastases were independently associated with a significantly higher probability of objective response. Hence, those features may help in recommending the best candidates for TEM-CAP.

To date, only one randomized study has compared TEM to TEM-CAP in patients with advanced PanNET, and its preliminary results were presented in 2018 [10]. In this phase II randomized multicenter study (E2211), 144 patients were randomized to receive TEM or TEMCAP. The patients in the TEM-CAP arm had prolonged median PFS (22.7 vs. 14.4 months, HR 0.58, 95\% CI 0.36$0.93, p=0.023)$ and OS (HR 0.41 [0.21-0.82], $p=0.012$ ) but, somewhat surprisingly, a similar ORR (33.3 vs. $27.8 \%, p=0.47)$. Nevertheless, this trial may have suffered 
from imbalance between the two arms, as the patients included in the TEM-CAP arm had PanNETs of a significantly lower grade (G2, 31.9 vs. $54.9 \%, p<0.01)$. Definitive results are pending, including a multivariate analysis with adjustment for tumor grade, as well as subgroup analyses depending on the MGMT status [10]. The results of the E2211 study are not directly comparable to those of our study, notably because the patients included in our study, as well as those in the study by Campana et al. [8], may have received more previous treatments. In fact, previous administration of cytotoxic chemotherapy appeared to be associated with lower TEM-CAP efficacy, maybe through a chemotherapy-induced selection of resistant tumor cells $[7,9]$. Finally, the rate of adverse events was significantly higher in the TEM-CAP arm of the E2211 study ( 44 vs. $22 \%, p=0.007$ ). Although retrospective data on tolerance should be interpreted with caution, we did not find any significant differences between TEM and TEM-CAP use. Especially the higher rate of asthenia in the TEM group is somehow surprising and might have been related to the selection of more fragile patients and/ or those with more advanced disease in this group.

The main limitations of our study are its retrospective setting and, subsequently, the absence of a calculation of subjects to include. In particular, such a retrospective, nonrandomized study cannot answer the question as to whether TEM-CAP is better than TEM alone; hence, our results must be interpreted with caution. Nevertheless, the use of a propensity score theoretically permits making the groups of patients comparable. Besides, the TEMCAP and the TEM group did not include the same number of patients, although a comparison of the same num- ber of patients matched for their propensity score yielded similar results.

Overall, although this study cannot answer the question as to whether the TEM-CAP combination is more effective than TEM alone, it suggests that TEM-CAP might not prolong PFS but might achieve a higher ORR. Tolerance to TEM-CAP might be similar or slightly impaired. Hence, TEM-CAP might be preferred when tumor shrinkage is the main therapeutic objective, particularly in a neoadjuvant setting, when surgery remains conceivable, or in case of a symptomatic and/or high tumor bulk. Conversely, TEM alone might be adequate in patients with an impaired performance status or in case of extrahepatic metastases.

\section{Statement of Ethics}

Data collection was performed following patient agreement according to the Declaration of Helsinki, and it was approved by the Institutional Review Board (IRB 00006477) of Paris University, France.

\section{Disclosure Statement}

L.d.M.: Ipsen (consulting), Novartis (research grant), and Pfizer (consulting); T.W.: Ipsen (consulting and research grant), Novartis (consulting and research grant), Keocyt (consulting), and Roche (research grant); P.d.B.: Genfit (stocks); O.H.: Ipsen, Novartis, and Pfizer (consulting); D.T.: Ipsen and Novartis (consulting); C.L.-B.: Novartis, Ipsen, AAA, and Pfizer (consulting); P.H.: Ipsen, Novartis, and Pfizer (consulting); P.R.: Ipsen, Novartis, AAA, and Keocyt (consulting); C.E., J.C., and V.R.: no conflicts of interest.

\section{References}

1 Dasari A, Shen C, Halperin D, Zhao B, Zhou $\mathrm{S}, \mathrm{Xu} \mathrm{Y}$, et al. Trends in the Incidence, Prevalence, and Survival Outcomes in Patients with Neuroendocrine Tumors in the United States. JAMA Oncol. 2017 Oct;3(10):1335-42.

2 Pavel M, O'Toole D, Costa F, Capdevila J, Gross D, Kianmanesh R, et al.; Vienna Consensus Conference participants. ENETS Consensus Guidelines Update for the Management of Distant Metastatic Disease of Intestinal, Pancreatic, Bronchial Neuroendocrine Neoplasms (NEN) and NEN of Unknown Primary Site. Neuroendocrinology. 2016; 103(2):172-85

3 Frilling A, Modlin IM, Kidd M, Russell C, Breitenstein S, Salem R, et al.; Working Group on Neuroendocrine Liver Metastases. Rec- ommendations for management of patients with neuroendocrine liver metastases. Lancet Oncol. 2014 Jan;15(1):e8-21.

4 Kulke MH, Hornick JL, Frauenhoffer C, Hooshmand S, Ryan DP, Enzinger PC, et al. O6-methylguanine DNA methyltransferase deficiency and response to temozolomidebased therapy in patients with neuroendocrine tumors. Clin Cancer Res. 2009 Jan; 15(1):338-45.

5 Strosberg JR, Fine RL, Choi J, Nasir A, Coppola D, Chen DT, et al. First-line chemotherapy with capecitabine and temozolomide in patients with metastatic pancreatic endocrine carcinomas. Cancer. 2011 Jan;117(2):268-75.

6 Fine RL, Gulati AP, Krantz BA, Moss RA, Schreibman S, Tsushima DA, et al. Cape- citabine and temozolomide (CAPTEM) for metastatic, well-differentiated neuroendocrine cancers: The Pancreas Center at Columbia University experience. Cancer Chemother Pharmacol. 2013 Mar;71(3):663-70.

7 Koumarianou A, Kaltsas G, Kulke MH, Oberg K, Strosberg JR, Spada F, et al. Temozolomide in Advanced Neuroendocrine Neoplasms: Pharmacological and Clinical Aspects. Neuroendocrinology. 2015;101(4):274-88.

8 Campana D, Walter T, Pusceddu S, Gelsomino F, Graillot E, Prinzi N, et al. Correlation between MGMT promoter methylation and response to temozolomide-based therapy in neuroendocrine neoplasms: an observational retrospective multicenter study. Endocrine. 2018 Jun;60(3):490-8. 
9 Kotteas EA, Syrigos KN, Saif MW. Profile of capecitabine/temozolomide combination in the treatment of well-differentiated neuroendocrine tumors. Onco Targets Ther. 2016 Feb;9:699-704.

10 Kunz PL, Catalano PJ, Nimeiri H, Fisher GA, Longacre TA, Suarez CJ, et al. A randomized study of temozolomide or temozolomide and capecitabine in patients with advanced pancreatic neuroendocrine tumors: a trial of the ECOG-ACRIN Cancer Research Group (E2211) [abstract 4004]. J Clin Oncol. 2018;36 15_suppl:36.

11 Klöppel G, Couvelard A, Hruban RH, Klimstra DS, Komminoth P, Osamura RY, et al Neoplasms of the neuroendocrine pancreas. In: WHO Classification of Tumours of the Endocrine Organs. 4th ed. Lyon: IARC Press; 2017. p. 210-39.

12 Therasse P, Arbuck SG, Eisenhauer EA, Wanders J, Kaplan RS, Rubinstein L, et al. New guidelines to evaluate the response to treatment in solid tumors. European Organization for Research and Treatment of Cancer, National Cancer Institute of the United States, National Cancer Institute of Canada. J Nat Cancer Inst. 2000 Feb;92(3):205-16.

13 Eisenhauer EA, Therasse P, Bogaerts J, Schwartz LH, Sargent D, Ford R, et al. New Response Evaluation Criteria in Solid $\mathrm{Tu}$ mours: revised RECIST guideline (version 1.1). Eur J Cancer. 2009 Jan;45(2):228-47.

14 Rosenbaum PR, Rubin DB. The Central Role of the Propensity Score in Observational Studies for Causal Effects. Biometrika. 1983; 70(1):41-55.

15 Austin PC. An Introduction to Propensity Score Methods for Reducing the Effects of Confounding in Observational Studies. Multivariate Behav Res. 2011 May;46(3):399-424.

16 Ekeblad S, Sundin A, Janson ET, Welin S, Granberg D, Kindmark H, et al. Temozolomide as monotherapy is effective in treatment of advanced malignant neuroendocrine tumors. Clin Cancer Res. 2007 May;13(10): 2986-91.

17 Maire F, Hammel P, Faivre S, Hentic O, Yapur L, Larroque B, et al. Temozolomide: a safe and effective treatment for malignant digestive endocrine tumors. Neuroendocrinology. 2009;90(1):67-72.

18 Welin S, Sorbye H, Sebjornsen S, Knappskog S, Busch C, Oberg K. Clinical effect of temozolomide-based chemotherapy in poorly differentiated endocrine carcinoma after progression on first-line chemotherapy. Cancer. 2011 Oct;117(20):4617-22.

19 Saif MW, Kaley K, Brennan M, Garcon MC Rodriguez G, Rodriguez T. A retrospective study of capecitabine/temozolomide (CAPTEM) regimen in the treatment of metastatic pancreatic neuroendocrine tumors (pNETs) after failing previous therapy. JOP. 2013 Sep;14(5):498-501.

20 Abbasi S, Kashashna A, Albaba H. Efficacy of capecitabine and temozolomide combination in well-differentiated neuroendocrine tumors: Jordan experience. Pancreas. 2014 Nov; 43(8):1303-5.

21 Peixoto RD, Noonan KL, Pavlovich P, Kennecke HF, Lim HJ. Outcomes of patients treated with capecitabine and temozolamide for advanced pancreatic neuroendocrine tumors (PNETs) and non-PNETs. J Gastrointest Oncol. 2014 Aug;5(4):247-52.

22 Schmitt AM, Pavel M, Rudolph T, Dawson H, Blank A, Komminoth P, et al. Prognostic and predictive roles of MGMT protein expression and promoter methylation in sporadic pancreatic neuroendocrine neoplasms. Neuroendocrinology. 2014;100(1):35-44.

23 Walter T, van Brakel B, Vercherat C, Hervieu V, Forestier J, Chayvialle JA, et al. O6-methylguanine-DNA methyltransferase status in neuroendocrine tumours: prognostic relevance and association with response to alkylating agents. Br J Cancer. 2015 Feb;112(3): 523-31.

24 Cives M, Ghayouri M, Morse B, Brelsford M, Black M, Rizzo A, et al. Analysis of potential response predictors to capecitabine/temozolomide in metastatic pancreatic neuroendocrine tumors. Endocr Relat Cancer. 2016 Sep;23(9):759-67.

25 Cros J, Hentic O, Rebours V, Zappa M, Gille $\mathrm{N}$, Theou-Anton N, et al. MGMT expression predicts response to temozolomide in pancreatic neuroendocrine tumors. Endocr Relat Cancer. 2016 Aug;23(8):625-33.

26 Ramirez RA, Beyer DT, Chauhan A, Boudreaux JP, Wang YZ, Woltering EA. The Role of Capecitabine/Temozolomide in Metastatic Neuroendocrine Tumors. Oncologist. 2016 Jun;21(6):671-5.

27 Crespo G, Jiménez-Fonseca P, Custodio A, López C, Carmona-Bayonas A, Alonso V, et al. Capecitabine and temozolomide in grade 1/2 neuroendocrine tumors: a Spanish multicenter experience. Future Oncol. 2017 Mar; 13(7):615-24.

28 Girot P, Dumars C, Mosnier JF, Muzellec L, Senellart H, Foubert F, et al. Short article: evaluation of O6-methylguanine-DNA methyltransferase as a predicting factor of response to temozolomide-based chemotherapy in well-differentiated metastatic pancreatic neuroendocrine tumors. Eur J Gastroenterol Hepatol. 2017 Jul;29(7):826-30.

29 Owen DH, Alexander AJ, Konda B, Wei L, Hemminger JA, Schmidt CR, et al. Combination therapy with capecitabine and temozolomide in patients with low and high grade neuroendocrine tumors, with an exploratory analysis of O6-methylguanine DNA methyltransferase as a biomarker for response. Oncotarget. 2017 Oct; 8(61): 104046-56

30 Raj N, Klimstra DS, Horvat N, Zhang L, Chou JF, Capanu M, et al. O6-Methylguanine DNA Methyltransferase Status Does Not Predict Response or Resistance to Alkylating Agents in Well-Differentiated Pancreatic Neuroendocrine Tumors. Pancreas. 2017 Jul;46(6): 758-63.

31 Fu D, Calvo JA, Samson LD. Balancing repair and tolerance of DNA damage caused by alkylating agents. Nat Rev Cancer. 2012 Jan; 12(2):104-20.

32 Murakami J, Lee YJ, Kokeguchi S, Tsujigiwa $\mathrm{H}$, Asaumi J, Nagatsuka H, et al. Depletion of O6-methylguanine-DNA methyltransferase by O6-benzylguanine enhances 5-FU cytotoxicity in colon and oral cancer cell lines. Oncol Rep. 2007 Jun;17(6):1461-7. 